\title{
On computational modeling of human- oriented knowledge in Ambient Intelligence
}

\author{
Tibor Bosse $^{\mathrm{a},{ }^{*}}$, Vic Callaghan ${ }^{\mathrm{b}}$ and Paul Lukowicz ${ }^{\mathrm{c}}$ \\ ${ }^{a}$ Department of AI, Vrije Universiteit Amsterdam, De Boelelaan 1081a, 1081 HV Amsterdam, The Netherlands \\ ${ }^{\mathrm{b}}$ Department of Computer Science, University of Essex, Wivenhoe Park, Colchester, Essex, CO4 3SQ, UK \\ ${ }^{\mathrm{c}}$ Embedded Systems Lab, Universität Passau, Innstraße 43, D-94032 Passau, Germany
}

\section{Background}

Recent developments within Ambient Intelligence (AmI) provide new possibilities to contribute to personal care, e.g. $[1,2,6,7]$. For example, our car may monitor us and warn us when we are falling asleep while driving. Similarly, the workspace of a technical worker may monitor the person's stress level, and provide support in case it is too high. As another example, an elderly person may wear a device that monitors his or her well-being and generates an action when a dangerous situation is noticed.

While such AmI applications can be initially based on the acquisition and fusion of sensory information, more intelligent applications need to depend on the availability of an adequate knowledge base for the analysis of human functioning. In recent years, scientific areas such as cognitive science, psychology, neuroscience and biomedical sciences have made substantial progress in providing increased insight in the physical and mental aspects of human functioning. Although much work still remains to be done, models have been developed for a variety of such aspects and the way in which humans (try to) manage or regulate them. From a biomedical angle, examples of such aspects are (management of) heart functioning, diabetes, and eating regulation disorders. From a psychological and social angle, examples are emotion regulation, attention regulation, addiction management, trust management, stress management, and criminal behavior management.

If models of human processes and their management are represented in a formal and computational format, and incorporated in intelligent agents in the human environment [5], then AmI applications are able to perform a more in-depth analysis of the human's functioning [4]. By endowing devices in the environment with explicit knowledge about humanoriented processes and with the capability to reason about the dynamics of such processes, an environment is created that has a better understanding of humans and how to support them, and is able to show a form of 'social intelligence' [3].

\section{About this thematic issue}

This thematic issue addresses computational modeling of human-oriented knowledge from disciplines such as psychology, social science, neuroscience and biomedical sciences, and the role such models may play within Ambient Intelligence. Following the published call, we received 13 contributions, from which, after a strict peer-review procedure, five papers have been selected for inclusion in this issue of JAISE. These five papers provide an illustrative overview of how various computational models of human-related processes can be applied in order to design more knowledgeable and effective AmI systems. The papers are briefly introduced below.

The first paper, by Aziz, Klein and Treur, addresses knowledge from clinical psychology. More specifically, a simulation model of unipolar depression is presented. Based on several personal characteristics and a representation of events (i.e., life events or daily hassles), this model can predict whether a human that recovered from a depression will fall into a relapse or recurrence. Moreover, by incorporating this model into an ambient agent system, the resulting system is able to reason about the state of the human and the effect of possible actions.

\footnotetext{
*Corresponding author. E-mail: tbosse@few.vu.nl.
} 
Several simulation experiments have been conducted to illustrate the functioning of the proposed system in different scenarios. In addition, an automated verification method is used to verify that the system satisfies a number of relevant properties.

The second paper, by Dovgan, Kaluža, Tušar and Gams, presents an AmI application in the security domain. Exploiting the Multi-Agent System paradigm, this paper presents a high-level security system that is able to verify a person's identity. This type of verification may be useful in various scenarios, varying from use of ATM machines to access of secure buildings. The presented system is able to verify a person's identity by processing data from various sensors (such as fingerprint readers and door sensors), and knowledge of the person's past behavior. The different types of data are processed by multiple agents, which communicate through a shared ontology. Eventually, classifications made by the different agents are integrated by the system in order to reach an overall decision about the person's identity.

The third paper, by Dybala et al., applies knowledge from psychology and sociology to Ambient Intelligence, in order to present a conversational (multi-agent) system for joke generation. The main idea of the paper is to improve performance of a chatterbot by endowing it with humor. A multi-agent system is presented, which integrates a conversational agent, a pun generator and an emotiveness analysis engine. The emotiveness analysis agent analyses users' utterances to decide whether it is appropriate to tell a pun. Depending on the results of this analysis, the agent either makes use of the pun generator or not. Two evaluation experiments point out that the humor-equipped system performs better than a baseline agent.

The fourth paper, by Mileo, Merico and Bisiani, focuses on the health care domain. To be specific, this paper tackles the problem of supporting independent living and well-being for people that live in their homes and have no critical chronic condition. The paper assumes the presence of a monitoring system equipped with a pervasive sensor network and a non-monotonic reasoning engine. However, the varied set of sensors that can be used for monitoring in home environments makes it difficult to provide a correct interpretation of collected data for a particular patient. For this reason, the authors introduce a logicbased context model for situation assessment, which is combined with logic programming techniques to reason about different pieces of knowledge of prevention.
The fifth paper, by Moran and Nakata, addresses the behavioral implications of ubiquitous monitoring. The authors claim that monitoring of humans (as assumed in many AmI applications) can often cause undesirable effects, such as an increase in stress. To investigate the impact of monitoring on users, they propose a dynamic model consisting of a series of factors believed to influence user behavior. Next, the model is augmented by the Theory of Planned Behavior, which enables it to explain and potentially predict undesirable effects in given scenarios. As the model has the characteristic of system dynamics, a preliminary simulation was carried out to examine the influence of each factor on one another, both directly and indirectly.

Although they address a variety of domains, the five papers collected in this thematic issue have in common that they propose systems which integrate detailed knowledge from human-directed disciplines (e.g., psychology, sociology, and biomedical sciences) with techniques to process this knowledge in an intelligent manner (e.g., simulation techniques, agent-based modeling, and logic-based approaches). We would like to emphasize that this combination is crucial in order to develop novel AmI systems that have an actual 'awareness' of the (physical and mental) states of humans, and are able to provide adequate support at the appropriate moment.

\section{References}

[1] E. Aarts, R. Collier, E. van Loenen, and B. de Ruyter (eds.) (2003). Ambient Intelligence. Proceedings of the First European Symposium, EUSAI 2003. Lecture Notes in Computer Science, vol. 2875, Springer Verlag, 2003, p. 432.

[2] E. Aarts, R. Harwig, and M. Schuurmans (2001). Ambient Intelligence. In: P. Denning (ed.), The Invisible Future, McGraw Hill, New York, pp. 235-250.

[3] E. Aarts and B. de Ruyter (2009). New research perspectives on Ambient Intelligence. In: Journal of Ambient Intelligence and Smart Environments 1, 2009, pp. 5-14.

[4] T. Bosse, M. Hoogendoorn, M.C.A. Klein, and J. Treur (2009). A Generic Architecture for Human-Aware Ambient Computing. In: E. Mangina, J. Carbo, and J.M. Molina (eds.), Agent-Based Ubiquitous Computing. Ambient and Pervasive Intelligence book series. Atlantis Press, 2009, in press.

[5] D.J. Cook (2009). Multi-agent smart environments. In: Journal of Ambient Intelligence and Smart Environments 1, 2009, pp. 51-55.

[6] G. Riva, F. Vatalaro, F. Davide, and M. Alcañiz (eds). (2005). Ambient Intelligence. IOS Press, 2005.

[7] W. Weber, J.M. Rabaey, and E. Aarts (2005). Ambient Intelligence. Springer Verlag New York, Inc. 